\title{
Les énoncés averbaux autonomes à deux termes comportent-ils un sujet syntaxique?
}

\author{
Florence Lefeuvre \\ Université Paris 3 - CLESTHIA-SYLED \\ florence.lefeuvre@univ-paris3.fr
}

\section{Introduction}

Parmi les énoncés averbaux autonomes à deux termes, certains suivent une structure liée :

(1) Stationnement interdit

(2) Bien avisé qui vous y trouverait (Mérimée ; ex. repris de Lefeuvre 1999 : 157)

(3) A chacun son destin

alors que d'autres suivent une structure segmentée ou détachée, avec des termes séparés par une virgule :

(4) Merveilleux, ces roses (ex. repris de Le Goffic 1993 : 514)

(5) Une belle ville, Toulouse (Bernanos; ex. repris de Lefeuvre 1999 : 159)

(6) Au diable, ta veillée, Colomba! (Mérimée; ex. repris de Lefeuvre 1999 : 197)

(7) Ici, le stylo !

Ces deux types d'énoncés sont basés sur un prédicat, participial ou adjectival ((1), (2), (4)), nominal (5), prépositionnel (6), adverbial (7). Dans ces structures, deux valeurs sémantiques dominent, celle de l'attribution (identification), en ((1), (2), (4), (5)), ou de la localisation ((3), (6), (7)) ${ }^{1}$. L'objet de cet article est de s'interroger sur les caractéristiques syntaxiques et sémantiques du terme qui accompagne le prédicat, un GN comme dans ces exemples (stationnement, son destin, ces roses, Toulouse, ta veillée, le stylo), ou bien un équivalent $\mathrm{du} \mathrm{GN}^{2}$ (qui vous y trouverait en (2)) que nous appellerons «terme $\mathrm{X}$ » (d'après Laurens 2011 qui parle de «terme $\alpha »$ ). Nous défendrons ici l'hypothèse que dans le premier groupe d'énoncés, ce GN (ou équivalent) se rapproche d'un sujet syntaxique et que dans le second groupe d'énoncés, il épouse certains traits du terme non lié, en structure disloquée, présent dans les énoncés verbaux tels que:

(8) C'est merveilleux, ces roses.

Pour autant, nous mettrons en évidence ce qui différencie le GN (ou équivalent) des énoncés ((1) à (3)) du sujet syntaxique des phrases verbales et le GN (ou équivalent) des énoncés ((4) à (7)) des dislocations des phrases verbales. Nous verrons que plusieurs critères permettent également de rapprocher ces deux types d'énoncés plutôt que de les opposer radicalement.

Après avoir expliqué en quoi ces énoncés sont autonomes et sont composés de deux termes, nous mettrons en évidence les ressemblances ou les distinctions entre d'une part le terme X des énoncés (1) à (7), et d'autre part le sujet d'une phrase verbale qui respecte la « servitude subjectale » (Hagège 1978:4), ce qui permettra de proposer une analyse pour ce terme, qui peut survenir dans une structure liée ((1) à (3)) ou non liée ((4) à (7)).

\footnotetext{
${ }^{1}$ On peut trouver d'autres valeurs comme celles de la possession Chacun son tour / Drôle de gueule, ce garçon (Pennac) : les phrases averbales, comme on le voit, ne sont pas glosables que par la phrase avec être.

${ }^{2} \mathrm{Cf}$. le point 3.3. pour la catégorisation de ce terme différent du prédicat.
} 


\section{Autonomie et composition de ces énoncés}

\subsection{Autonomie}

Nous considérerons, d'après Lefeuvre 1999, toute structure syntaxique comme autonome dans la mesure où elle repose sur un prédicat assorti d'une modalité d'énonciation.

\subsubsection{Les exemples du premier type}

Les exemples du premier type sont de deux sortes.

Tout d'abord, certains de ces exemples comportent des énoncés de type générique, comme $A$ chacun son destin en (3) ou :

(9) Heureux les simples d'esprit !

ou bien des énoncés renvoyant à un sujet indéfini (qui vous y trouverait en (2)). Leur modalité est l'assertion, qui rappelle celle délivrée dans les proverbes ou les maximes.

Ensuite, les autres exemples suivent le schéma "Nom sans déterminant ${ }^{3}+$ participe passé", en (1) et :

(10) Impression annulée (Imprimante)

(11) Texte de L. non encore parvenu (Note)

(12) Lucette.- Mais, prends garde ! Si tu me laisses franchir le seuil de cette porte, tu ne me reverras jamais !

Bois-d'Enghien. - Marché conclu ! (Feydeau)

ou encore, "Nom sans déterminant + adjectif ou groupe prépositionnel" :

(13) Code bon (Carte bleue)

(14) Départ imminent (Tramway)

(15) Pour votre sécurité, trafic routier sous video surveillance (Panneau)

(16) « Sabre au clair donc» (Stendhal; ex. repris de Lefeuvre 1999: 195)

(17) Rats en campagne aussitôt (La Fontaine; ex. repris de Lefeuvre 1999 : 209)

Ils rassemblent, pour ce qui concerne le terme $X$, des caractéristiques qui s'opposent nettement à celles du terme $\mathrm{X}$ des énoncés de type 2 . Le problème de certaines de ces structures, c'est qu'elles ne semblent pas énonçables, notamment celles qui figurent sur des panneaux, telles que (1) Stationnement interdit. L'absence de prise en charge explicite d'un locuteur (cf. Delorme 2004a et 2004b:345), propre à l'absence de verbe, est renforcée par la difficulté d'énoncer de tels segments. On peut se demander dès lors s'il y a bien assertion. Nous pouvons les considérer comme phrastiques grâce à trois éléments :

i) Ils se trouvent dans des types de corpus variés : ils peuvent bien sûr se trouver inscrits sur des panneaux (1), mais aussi sur des affichages électroniques ((10), (15)), dans des notes récapitulatives (11); ils peuvent en fait apparaître dans toutes sortes de textes, issus par exemple de la littérature, dans des conversations fictives, dramaturgiques (12) ou romanesques (16) ou même dans des récits (une fable en (17)).

ii) Ces structures peuvent se décliner avec une autre modalité, lorsque la situation d'énonciation le permet, notamment lorsqu'elles sont insérées dans le corps d'un texte :

(18) Rats en campagne aussitôt ? Il semble que oui.

En (16), la modalité est injonctive. Nous considérerons donc que ces énoncés sont assertés soit avec un locuteur standard, identifié (Bois-d'Enghien en (12)), soit avec un locuteur qui correspond à une institution, ce qui rend particulières les conditions d'énonciation de ces structures.

\footnotetext{
${ }^{3}$ Nous préférons ne pas considérer que ces structures comportent le déterminant $\varnothing$ parce qu'elles ne peuvent pas commuter avec des structures comportant un déterminant : * Le stationnement interdit.
} 
iii) Enfin leur valeur prédicative semble bien réelle, comme le montre la possibilité d'avoir des marqueurs de prédication, des intensifs ((19), (20)), l'adverbe de négation non (21), accompagnée en (22) de l'adverbe aspectuel encore :

(19) Entrée exclusivement réservée aux groupes (Théâtre La Cachette, $13^{\mathrm{e}}$ arrondissement)

(20) Détaxe sous douane définitivement fermée

(21) Saisie erronée : informations non enregistrées (Antarès)

(22) Texte de L. non encore parvenu (Note).

\subsubsection{Les exemples du second type}

En ce qui concerne les énoncés du second type ((4) à (7)), ils acceptent les trois modalités d'énonciation, l'assertion ((1) à (5)), l'injonction ((6), (7)), l'interrogation :

(23) Ingrats, les amis du président? (Le Nouvel Observateur)

Le prédicat, quant à lui, se reconnaît dans la possibilité qu'il a d'accueillir des «marqueurs de prédication » (cf. Lefeuvre 1999 et Behr \& Lefeuvre 2005) tels que les adverbes de négation :

(24) Pas mécontent de sa formule le p'tit type (Queneau)

C'est généralement un terme subjectif qui se distingue par son caractère évaluatif ou affectif (cf. Lefeuvre 1999), mis régulièrement en première position. Le terme $X$, non prédicatif, est quant à lui le plus souvent défini et spécifique, renvoyant à un référent identifiable. Il prend plus rarement une valeur générique, mettant alors le prédicat en seconde position, en raison d'une valeur plus classifiante :

(25) Ainsi la mort, la vie, l'éternité, choses fort simples pour qui aurait les organes assez vastes pour les concevoir... (Stendhal)

(26) L'homme, une espèce à découvrir (titre)

\subsection{Un ou deux termes}

Les structures que nous venons d'évoquer sont-elles constituées d'un ou de deux termes? La présence de deux termes apparaît comme évidente pour des énoncés segmentés tels que (4) ou même pour des énoncés non segmentés où le prédicat est en première position (Bien avisé qui vous y trouverait) (2). Qu'en est-il pour des énoncés tels que Stationnement interdit (1) où, selon nous, c'est un prédicat qui apparaît en deuxième position et non une épithète ? Avec cet ordre (cf. 2.3.), il peut être plus difficile de distinguer deux groupes avec deux rôles syntaxiques différents au sein de la phrase. Nous allons pourtant voir que c'est bien le cas.

Le premier terme correspond généralement à une nominalisation ${ }^{4}$. On peut trouver, selon le classement opéré par Vendler (1957/1967), des noms renvoyant à un état (stationnement en (1)), une activité :

(27) Circulation sur bande d'arrêt d'urgence interdite (autoroute)

un accomplissement :

(28) Traversée du pont de l'Arche interdite

ou encore un achèvement :

(29) Accès interdit aux cyclomoteurs, cycles, chiens (square Héloïse et Abélard, Paris

$\left.13^{\mathrm{e}}\right)$

(30) Paiement accepté

\footnotetext{
${ }^{4}$ Cf. Lefeuvre et Nicolas 2004 pour l'étude aspectuelle des nominalisations prédicatives.
} 
La nominalisation a pu donner lieu à un sens non processuel, comme le mot entrée qui désigne en (19) un lieu et non l'action d'entrée. On peut trouver des noms qui ne sont pas issus d'une nominalisation. Certains désignent un lieu :

(31) Route contrôlée par radar (panneau),

d'autres des actants renvoyant à des individus ou des objets :

(32) Parents mobilisés pour la qualité d'enseignement pour tous (école Jeanne d'Arc, Paris $\left.13^{\mathrm{e}}\right)$

Dans tous ces exemples, le nom n'est pas précédé d'un déterminant : c'est le contexte, situationnel ou linguistique, qui fournit les indications nécessaires afin de déterminer le référent du nom en question. Généralement cela s'établit grâce au contexte situationnel : la localisation des panneaux ou écriteaux divers permet de renvoyer le nom en question à un référent précis. En (30), paiement renvoie au paiement effectué par quelqu'un à tel moment et dans tel lieu. La dénotation du déverbal paiement s'établit en fonction de la situation d'énonciation (ici particulière puisqu'il s'agit d'une machine qui délivre le message). On note ainsi une dimension déictique de ces structures.

Parfois le calcul de la référence se fait grâce au contexte linguistique, comme en (12) : dans Marché conclu, le mot marché reprend ce qui a été dit précédemment. Il a une portée résomptive, dans le sens où il renvoie à une prédication (cf. Lefeuvre 2007 : 146-147, d'après Maillard 1974 : 57), parfois avec une valeur caractérisante. C'est le cas également de cet énoncé :

(33) Il accueille les femmes par un baise-main délicieusement vieille France. Sur la défensive, pourtant. Ne va-t-on pas encore lui ressortir les affaires de la mairie de Paris? Essayer de lui faire dire du mal de Sarkozy? Peine perdue. Parler de politique, il s'y refuse. De lui, Il y répugne. (Le Nouvel Observateur, 26 février 20094 mars 2009)

Le mot peine résume les prédications verbales énoncées précédemment: lui ressortir les affaires de la mairie de Paris, essayer de lui faire dire du mal de Sarkozy.

Le second terme est généralement un participe passé à valeur résultative : interdit, réservée, accepté (mais pas contrôlée en (31), sans doute à cause de la présence du complément d'agent par radar). On peut s'interroger sur cette prépondérance des participes passés : leur aspect résultatif entre en opposition avec la situation (état ou action) en cours (au moins potentiellement), dénotée par la nominalisation. Cette différence aspectuelle favorise la lecture de ces énoncés comme deux groupes plutôt que comme un seul groupe.

Des indices syntaxiques montrent également qu'il s'agit bien de deux termes distincts. Le fait que les marqueurs de prédication mentionnés ci-dessus se trouvent devant le participe et non devant la nominalisation :

(34) Stationnement strictement interdit

(35) * Strictement stationnement interdit

nous indique qu'il ne s'agit pas d'un seul ensemble mais que ces énoncés comportent deux termes pourvus de fonctions différentes. D'autres indices syntaxiques nous montrent qu'il s'agit bien de structures à deux termes.

i) Un complément de nom en de ne peut être introduit qu'après la nominalisation et non après interdit, ce qui montre que interdit n'est pas une épithète mais un prédicat :

(36) Accès du parc interdit

(37) *Accès interdit du parc

C'est ce que l'on trouve dans les exemples suivants :

(38) Circulation sur bande d'arrêt d'urgence interdite (Panneau)

(39) Traversée du pont de l'Arche interdite (Panneau autoroute A 13) 
Dans ce nouvel exemple :

(40) Traversée fréquente de grands animaux (Panneau)

la présence du complément de nom après fréquente montre que cet adjectif est épithète et non prédicatif ${ }^{5}$. En revanche, l'ordre des termes adj $+\mathrm{N}$ (lorsqu'il est possible) implique un statut épithétique de l'adjectif :

(13') Bon paiement

ii) Le participe reçoit ses propres compléments :

(41) Vol terminé à 11 h10 (Ecran d'aéroport)

(42) Stationnement limité de $9 \mathrm{~h}$ à $18 \mathrm{~h}$ (Panneau)

iii) Enfin, lorsque l'énoncé comporte un adjectif et un participe passé :

(43) Piste cyclable déviée dans la circulation générale (Boulevard Vincent Auriol, août 2008)

c'est le participe passé qui est senti comme prédicatif plutôt que l'adjectif qui joue le rôle d'une épithète :

(44) *Piste entièrement cyclable déviée dans la circulation générale

(45) Piste cyclable entièrement déviée dans la circulation générale.

Cela dit, certaines de ces expressions apparaissent comme figées (Marché conclu en (12); Peine perdue en (33)) ; dans ce cas-là, il est plus difficile d'apporter des modalisations au prédicat :

(46) Lucette.- Mais, prends garde ! Si tu me laisses franchir le seuil de cette porte, tu ne me reverras jamais!

Bois-d'Enghien. - Je ne suis pas d'accord. ? Marché pas conclu du tout

En outre, comme souvent pour les structures averbales, les conditions d'énonciation peuvent impliquer un autre type d'analyse. Par exemple, il existe des ensembles "Nom sans déterminant + participe passé" qui composent un thème, sans valeur prédicative :

(47) Femmes battues

Elles témoignent (Ouest France mardi 26 août 2012)

\section{Les critères de la « servitude subjectale » (Hagège 1978)}

Voyons à présent si les différents critères de « la servitude subjectale » qui permettent de définir le sujet dans les phrases verbales :

Ce qui définit, dans les termes les plus clairs, la servitude subjectale, c'est un phénomène formel, l'accord, qui en reflète un autre, le caractère indispensable auquel s'en ajoute, le plus souvent, un troisième, la position avant le prédicat (Hagège $1978: 14)$

sont remplis par les termes non prédicatifs des énoncés du premier type (1) et des énoncés du second type (4).

\subsection{L'accord}

Dans les énoncés du premier type (1) :

(48) Traversée du Pont de l'Arche interdite.

(49) Heureuses celles qui découvrent le Seigneur

\footnotetext{
${ }^{5}$ Pour une réflexion plus générale sur la fonction prédicative (le prédicat, l'attribut ou l'apposition), voir par exemple Riegel et al. 2000.
} 
l'accord est impossible à ne pas effectuer :

(50) *Traversée du Pont de l'Arche interdit.

(51) *Heureux celles qui découvrent le Seigneur

Voyons ce qu'il en est pour les énoncés du second type. Il est parfois signalé que l'accord n'est pas indispensable (Le Goffic 1993, Lefeuvre 1999, Deulofeu 2003, Laurens 2011). Nous nuancerons ce point. L'absence d'accord est possible si le prédicat peut caractériser une situation plutôt que l'objet proprement dit dénoté par le GN défini. C'est le cas de l'exemple (4) :

(4) Merveilleux, ces roses ! (ex. repris de Le Goffic 1993 : 514)

où l'adjectif renvoie à des propriétés liées aux roses : ces roses sont belles, sentent bon etc. Il y a comme une décatégorisation du GN qui rappelle ce qui se passe dans les phrases verbales avec le démonstratif $c$ ', grâce à son sémantisme du non catégorisé (Corblin 1987, Kleiber 1994 : 75) :

(52) C'est merveilleux, ces roses !

Dans l'énoncé :

(53) Merveilleuses, ces roses !

merveilleuses caractérise uniquement le référent ces roses. La position en second du prédicat n'implique pas le caractère obligatoire de l'accord :

(54) Ces roses, merveilleux

à partir du moment où une caractérisation des propriétés de ces roses est possible. Certains énoncés ne permettent pas d'évoquer ces propriétés. Ainsi, dans cet exemple :

(55) Confisquée, la robe ! (Feydeau ; ex. repris de Lefeuvre 1999 : 148)

(56) *Confisqué, la robe !

confisquée ne peut pas être prédiqué des propriétés de la robe mais seulement de l'objet la robe, d'où le caractère obligatoire de l'accord. Il nous semble malaisé également de ne pas effectuer l'accord dans l'exemple suivant :

(57) Durs, rebelles et carnassiers, ces Lestrygons ! (Le Nouvel Observateur)

(58) *Dur, rebelle et carnassier, ces Lestrygons !

ou encore dans cet énoncé :

(59) Brillants, ces étudiants.

(60) *Brillant, ces étudiants.

Mais un autre type de GN non lié ou de prédicat rend l'accord facultatif :

(61) Brillant, cette remarque.

(62) Incroyable, ces Lestrygons.

lorsque la combinaison de l'adjectif avec le GN défini permet de suggérer autre chose que le référent du GN proprement dit; en (61), brillant renvoie aux propriétés de cette remarque : par exemple celle-ci vise juste, explique bien la situation, est digne de son auteur etc. ; en (62), incroyable peut être prédiqué de ce que sont ou font ces Lestrygons : ces géants sont féroces, dévorent des hommes etc.

Nous retiendrons donc que, selon qu'il y a accord ou non, la prédication visée n'est pas la même. Les énoncés (4) et (53) sont différents et ne correspondent pas à la même prédication. Nous différencierons ces cas de figure où l'accord peut ne pas être effectué des expressions où l'absence d'accord signale un figement. Il en est ainsi de bon, dans à quoi bon ${ }^{6}$ :

\footnotetext{
${ }^{6}$ Pour les autres expressions figées où l'accord n'est pas établi (avec ci-joint par exemple), cf. Grevisse, $1988, \S 248$.
} 
(63) A quoi bon ces perpétuels appels à l'opinion, quand l'opinion ne réagit plus et que le gouvernement peut se permettre de n'en plus tenir compte? (Le Nouvel Observateur)

Bon, quel que soit l'exemple, ne s'accorde jamais dans cette expression :

(64) *à quoi bons ces perpétuels appels à l'opinion?

\subsection{Le caractère indispensable}

Pour les énoncés du premier ensemble (1), il est difficile voire impossible de supprimer le nom ${ }^{7}$ :

(65) Traversée du Pont de l'Arche interdite

(66) Heureux les simples d'esprit

en l'occurrence Traversée du Pont de l'Arche et les simples d'esprit :

(67) * interdite.

(68) *Heureux

Dans les exemples tels que (65), aucun signe de ponctuation n'est possible entre les deux termes :

(69) Chaussée glissante

(70) *Chaussée : glissante

Néanmoins, le deux points pourrait survenir dans certains corpus, comme celui des indications météorologiques :

(71) Température : variable

Le prédicat (variable) appartient alors à un paradigme de caractérisations possibles : saisonnière, stable, en hausse etc. Ce type d'indications est fourni avec régularité. En revanche, sur les panneaux comme en (65) ou (69), on ne s'attend pas à ce qu'il y ait d'autres propriétés concernant la traversée du Pont de l'Arche ou la chaussée, ce qui rend impossible l'utilisation du deux points.

Pour les énoncés du second type (4 à 7), il est souvent précisé (par exemple dans Le Goffic 1993, Lefeuvre 1999, Laurens 2011) que le terme différent du prédicat est omissible. En fait, cela dépend de la situation d'énonciation ou du discours où se trouve inséré l'énoncé en question : l'objet dénoté par le GN est-il accessible à la connaissance de chacun sans passer par sa formulation ? Sans ce terme, on peut ne pas bien comprendre à quoi renvoie le prédicat. Ainsi dans l'exemple suivant :

(72) Ce n'est pas la première fois que Ségolène Royal éprouve la désagréable impression d'être «surveillée ». Pendant la campagne présidentielle, qui avait vu les RG s'intéresser de près à un de ses conseillers, l'écologiste Bruno Rebelle, elle s'était émue que plusieurs ordinateurs portables disparaissent dans son entourage. [...]. Plus surprenant encore : fin juillet 2007, tandis que quelqu'un dérobait un ordinateur dans son appartement, un autre portable disparaissait le même week-end dans les bureaux d'un de ses plus proches soutiens.

Alors, paranoïaque, Ségolène Royal ? «Un peu, parfois, reconnaît Julien

Dray, qui la connaît bien. [...]. (Le Nouvel Observateur, 17-23 juillet 2008)

il nous semble difficile d'omettre Ségolène Royal:

(73) ? Alors, paranoïaque ?

En (73), l'adjectif, isolé entre deux ponctuations fortes, renvoie au contexte de gauche (cf. Lefeuvre 2007), mais dans cet exemple, aucun nom ni pronom de la phrase précédente ne pourrait être caractérisé par l'adjectif paranoïaque. Et Ségolène Royal ne forme pas un référent suffisamment saillant pour que

\footnotetext{
${ }^{7}$ Notons cependant que la suppression est envisageable si le participe interdit est au masculin et si un panneau évoque par une représentation graphique l'action à éviter.
} 
tout lecteur comprenne bien que l'adjectif paranö̈aque lui revient. Un nom conviendrait mieux et caractériserait alors les énoncés précédents :

(74) Alors, paranoïa ?

Dans la phrase verbale :

(75) Alors, elle est paranoïaque ?

la présence du pronom elle fournit un indice supplémentaire pour récupérer le référent. Pour cette raison, il est plus difficile de supprimer le terme non prédicatif (Ségolène Royal en (72), dans les structures averbales que dans les structures verbales segmentées.

En outre, toujours en ce qui concerne les énoncés du second type (4 à 7), la solidarité entre les deux termes peut être plus forte. Il existe des énoncés averbaux où aucune virgule ne les sépare, lorsque le prédicat est en seconde position :

(76) Royal victime de la presse ? On connaît la propension de la présidente de Poitou-Charentes à se poser en martyre des médias. (Le Nouvel Observateur)

mais aussi lorsqu'il se trouve en première position, comme en (24) ou surtout avec des prédicats locatifs (non caractérisants) :

(77) Eh bien, cousin, à quand le mariage? (Musset; ex. repris de Lefeuvre $1999: 202)$

(78) - A cette nuit les details, ajouta-t-elle en riant (Stendhal ; ex. repris de Lefeuvre $1999: 143)$

(79) Loin de moi cette idée !

L'inversion des termes paraît alors difficile :

(80) ?Le mariage, à quand ?

(81) ? Les détails, à cette nuit.

(82) ? Cette idée, loin de moi.

\subsubsection{Position contrainte}

Les énoncés du premier type ((1) à (3)) se caractérisent par la position contrainte du GN. Les énoncés génériques ou généralisants n'acceptent pas l'inversion des termes :

(83) *Les pauvres heureux

Et pour ceux du type (1) (Stationnement interdit), c'est l'antéposition qui est la règle. L'absence de déterminant rend impossible l'ordre inverse :

(84) Stationnement interdit

(85) *Interdit, stationnement.

Pour les énoncés du second type ((4) à (7)), il est possible d'inverser les termes (4) mais la position n'est pas si libre que cela. Tout d'abord on observe une tendance inverse à celle des «dislocations » des phrases verbales. Si l'on se base sur le corpus donné dans Blasco-Dulbecco 1999 sur les dislocations dans les phrases verbales, elles sont nettement plus importantes avant le verbe qu'après le verbe, que le corpus soit écrit ou oral :

Il faut noter que $69,25 \%$ des dislocations à l'oral se situent avant le verbe et $30,75 \%$ après le verbe.

A l'écrit, 67, $92 \%$ des dislocations sont avant le verbe et $32,08 \%$ après le verbe. (Blasco, 1999 : 91) 
Dans la phrase averbale en revanche, la tendance est inverse. Une quantification de plus grande ampleur reste à faire, mais sur les cinq romans passés en revue ${ }^{8}$, on observe deux tiers d'exemples où le GN non prédicatif et non lié est en deuxième position (comme dans les exemples 4 à 7 ) et un tiers d'exemples où le GN non prédicatif et non lié est en première position, comme dans cet exemple :

(86) ... Ainsi la mort, la vie, l'éternité, choses fort simples pour qui aurait les organes assez vastes pour les concevoir... (Stendhal)

Cette position à droite n'est pas anodine. Un énoncé composé en premier d'un terme averbal et en second d'un GN défini ((4) à (7)) offre d'emblée une lecture prédicative de ce premier terme. Avec l'ordre inverse :

(87) Le Parisien, près de chez vous, proche de vous ! (Publicité)

(88) - Oui, une qui flaire comme baume! elle est auprès de mon pupitre sur le comptoir. La lettre de madame de Bargeton mêlée aux bocaux de la pharmacie !

Lucien s'élança dans la boutique (Balzac ; ex. repris de Lefeuvre 1999 : 171)

le participe ou l'adjectif qui caractérise un nom, qui plus est sans virgule, est lu d'emblée comme un terme appartenant au GN. L'ensemble composant un groupe défini est alors vu comme un sujet ou un thème en attente du prédicat, ce qui pourrait donner pour $(88)$ :

(89) La lettre de madame de Bargeton mêlée aux bocaux de la pharmacie, c'est un scandale!

Ainsi peut-on soutenir que la position des termes dans les énoncés averbaux autonomes du second type obéit à une contrainte de type syntaxique.

Voyons à présent les conséquences du respect ou du non respect de la servitude subjectale.

\section{Sujet ou terme périphérique ?}

Vu les données, quelles appellations serait-il possible de proposer, dans ces énoncés averbaux autonomes, pour le terme différent du prédicat?

\subsection{Sujet syntaxique ?}

L'appellation de « sujet syntaxique » pourrait concerner les énoncés du premier groupe (1 à 3). En effet, les trois paramètres - accord, caractère indispensable, position contrainte — sont clairement respectés, ce qui permet d'apparenter ce terme à un sujet de type syntaxique.

Cela dit, il ne s'agit pas du même type de sujet que dans une phrase verbale puisqu'un groupe comme Traversée du pont de l'Arche ne peut pas être remplacé par un pronom clitique :

(90) Traversée du pont de l'Arche interdite

(91) *Elle interdite.

Seul serait possible le pronom disjoint dans une structure comprenant un détachement :

(91') Elle, interdite ?

(91') Elle, interdite !

ce qui donne pour (57):

(92) Durs, rebelles et carnassiers, eux !

\footnotetext{
${ }^{8}$ N'ont été considérés que les exemples où le prédicat est un GN ou un adjectif. Les romans passés en revue sont les suivants : Journal d'un Curé de Campagne (Bernanos), Le Rouge et le Noir (Stendhal), Tartarin de Tarascon (Daudet), Thérèse Desqueyroux (Mauriac), Zazie dans le Métro (Queneau).
} 


\subsection{Terme périphérique ?}

Pour les énoncés du second groupe ((4) à (7)), le terme non prédicatif a été régulièrement assimilé à un terme disloqué : cf. Le Goffic 1993 : 514 («construction détachée (disloquée ») / «terme thématisé, autonome»), Lefeuvre 1999:185 («structure disloquée»), et plus récemment, Deulofeu 2003: 137 ( «terme détaché »), Laurens $2011: 48$ (« syntagme disloqué droit », « en périphérie »).

On voit ce qui justifie ces diverses appellations : dans les énoncés de ce type ((4) à (7)), lorsque les deux termes sont séparés par une virgule, ils sont nettement moins solidaires que pour les énoncés du premier groupe tels (1) Stationnement interdit. Dans ce cas, le terme non prédicatif ne peut pas être considéré comme un terme essentiel, proche d'un sujet syntaxique.

Considérer comme périphérique le terme différent du prédicat permettrait de rapprocher les énoncés du second groupe, d'énoncés composés uniquement du prédicat (cf. Deulofeu 2003 : 137) :

(93) A Paris, au sol, il se sent à l'étroit. Claustrophobe? «En ville, on vit dans des boîtes rectangulaires, empilées les unes sur les autres : chambres, bureaux, cafés...

Alors, il faut sans cesse ruser avec ce sentiment d'incarcération », explique l'écrivain. (Le Nouvel Observateur, mars 2008)

Mais dès que l'on examine les exemples attestés en discours, les emplois des énoncés à deux termes ou à un terme ne sont pas toujours interchangeables (cf. ici (72) et (73)) et s'avèrent en fait d'un emploi en discours différent.

D'autres raisons, vues en 2, montrent que les appellations de «périphérique » ou de «terme autonome » marquent un écart trop important par rapport au prédicat. Rappelons : la présence ou l'absence d'accord qui marquent des prédications différentes et qui ne signifient donc pas la possibilité de supprimer le GN sans conséquence ; la solidarité entre les termes plus ou moins forte, avec une virgule qui n'apparaît pas systématiquement; la position à droite du prédicat, appelée par ce type de structure averbale. Ces appellations ne peuvent se justifier que dans la prédication averbale où l'accord n'est pas marqué, où le caractère non lié est net comme dans Merveilleux, ces roses. Pour ce qui est des appellations de " disloqué » ou de " détaché », elles supposent que la structure standard est liée, ce qui n'est pas le cas pour les structures averbales qui comportent parfois de façon obligatoire une virgule et qui ne suivent pas le schéma lié des phrases verbales.

Nous pouvons ajouter une raison de type sémantique : les termes de "périphérique » ou d' "autonome » masquent le lien sémantique qui existe entre le terme non prédicatif et le prédicat averbal. Le terme différent du prédicat est caractérisé, identifié ou localisé par le prédicat. Cela ne peut pas être un GN défini à valeur temporelle comme ce 18 décembre 2008 dans l'exemple suivant :

(94) Quel beau monde, ce 18 décembre 2008, autour de « Monsieur Lu »! (Le Nouvel Observateur)

Ce GN se différencie d'un groupe tel que ces roses en (4) ou ces Lestrygons en (52) en ce qu'il n'est pas caractérisé par le prédicat. C'est ici un circonstant temporel.

\subsection{Sujet sémantique ?}

C'est pourquoi, nous préférons proposer l'appellation de « sujet sémantique » (cf. Muller 2002) renvoyant à ce qui est caractérisé, identifié ou localisé par le prédicat. Si l'on se place sur un plan informationnel, cet élément détaché se distingue par « une fonction de repérage référentiel » (Prévost 2003) par rapport au prédicat averbal, qui lui se trouve focalisé (Wilmet, 2003 : § 586). Il accèderait au statut de « topique », « ce à propos de quoi il est pertinent de dire quelque chose » (Prévost 2003), dans la prédication suivante. Cela permet de dégager deux pôles, l'un où le terme différent du prédicat fonctionne comme un constituant proche d'un sujet syntaxique (1) et l'autre où il se comporte comme un terme périphérique (Merveilleux, ces roses (4)). Entre ces deux pôles, se trouvent des énoncés où le terme non prédicatif se caractérise par un statut intermédiaire, ne possédant pas tous les attributs du sujet syntaxique ni ceux d'un terme périphérique.

Ce terme différent du prédicat possède une caractéristique habituelle pour un sujet : c'est un GN, défini la plupart du temps, ou un équivalent du GN. Pour les rares exemples qui comportent un groupe prépositionnel (et non un GN) : 
(95) Une vraie porcherie, chez lui (ex. repris de Laurens 2011 : 59)

on peut considérer qu'il s'agit en fait de noms en discours :

(96) Une vraie porcherie, son chez lui.

Effectivement, tous les groupes prépositionnels ne peuvent pas se trouver dans ce type de construction. Certains locatifs possibles dans une phrase avec être:

(97) Il est chez lui / dans sa maison

ne peuvent pas survenir dans une structure averbale segmentée :

(98*Une vraie porcherie, dans sa maison

ou bien l'énoncé prendrait un sens existentiel :

"Il y a une vraie porcherie, dans sa maison". ou encore dans sa maison serait vu comme un circonstant :

“c'[ce que j'ai vu] est une vrai porcherie, dans sa maison".

Ce faisant, et contrairement à certains auteurs (Deulofeu 2003), nous n'établissons pas de rupture définitive entre les énoncés du premier type et les énoncés du second type, la raison principale étant que la solidarité entre les termes suit un continuum : parfois elle est faible, parfois elle est forte, parfois nous avons des cas intermédiaires.

\section{Conclusion}

Les énoncés averbaux autonomes à deux termes tels que Stationnement interdit s'avèrent différents de ceux du type Durs, rebelles et carnassiers, ces Lestrygons! (Le Nouvel Observateur). La solidarité des termes dans le premier type d'exemples permet de voir dans ces énoncés la présence d'un constituant apparenté à un sujet syntaxique. Dans le second type d'exemples, si le GN défini se rapproche de l'élément non lié présent dans les phrases verbales (Ils sont durs, ces Lestrygons), il s'en éloigne par le rôle structurel qu'il remplit dans ce type d'exemple. La gradation du caractère segmenté de ces structures montre que le caractère périphérique du terme non prédicatif n'est pas systématique et incite à proposer le terme plus général de "sujet sémantique". Il existe un continuum marqué par deux pôles, l'un où la structure est clairement liée et l'autre où la structure est clairement segmentée ; entre les deux, il existe toute une série d'énoncés intermédiaires.

\section{Références bibliographiques}

Behr, I. \& Lefeuvre, F. (2005). La configuration GN GN : comment reconnaître le prédicat ?. in Behr, I. \& François, J. dir., Les Constituants prédicatifs et la diversité des langues, Mémoires de la Société linguistique de Paris, tome $X I V$, Louvain : Peeters, 199-21.

Blasco-Dulbecco, M. (1999). Les Dislocations en français contemporain. Paris : Champion.

Corblin, F. (1987). Ceci et cela comme formes à contenu indistinct. Langue française, 75, 75-93

Delorme, B. (2004a). Les énoncés nominaux dans la fiction contemporaine de langue anglaise, implications sémantiques et pragmatiques de la prédication averbale, thèse de doctorat, Paris 4-Sorbonne.

Delorme, B. (2004b). Prédication averbale et rupture de repérages. In Lefeuvre, F. dir., La phrase averbale: délimitation et caractéristiques, Verbum XXVI, 4, 343-453.

Deulofeu, J. (2003). Lontan dei occhi, lontan del cuore : les énoncés averbaux sont des énoncés comme les autres. In La rime et la raison : hommage à Benô̂t de Cornulier, Paris : Champion, 123-138.

Grevisse, M. (1988). Le bon Usage. Paris-Gembloux : Duculot. 
Hagège, C. (1978). Du thème au thème en passant par le sujet. Pour une théorie cyclique. La Linguistique, 14, 2, 338.

Kleiber, G. (1994). Anaphores et pronoms. Louvain-la-Neuve : Duculot.

Laurens, F. (2011). Analyse des énoncés averbaux prédicatifs autonomes. In Lefeuvre, F. et Behr, I. dir., Les énoncés averbaux autonomes entre grammaire et discours. Paris :Ophrys, 47-64.

Lefeuvre, F. et Nicolas, D. (2004). La phrase nominale existentielle et la distinction aspectuelle télique / atélique. Revue de sémantique et pragmatique, 14, 101-119.

Lefeuvre F. (1999). La phrase averbale en français. Paris, L’Harmattan.

Lefeuvre, F. (2007). Le segment averbal comme unité syntaxique textuelle. In Charolles, M., Fournier, N., Fuchs, C., Lefeuvre, F. dir., Parcours de la phrase : Ophrys, 143-158.

Le Goffic P. (1993). Grammaire de la phrase française. Paris, Hachette.

Maillard, M. (1974). Essai de typologie des substituts diaphoriques. Langue française, 21, 55-71.

Moignet, G. (1981). Systématique de la Langue française. Paris : Klincksieck.

Muller, C. (2002). Les Bases de la syntaxe. Passac: Presses universitaires de Bordeaux.

Prévost S. (2003). Détachement et topicalisation : des niveaux d'analyse différents. Cahiers de Praxématique, 40, $97-$ 126. http://halshs.archives-ouvertes.fr/docs/00/34/99/00/PDF/prevost-biblio5.pdf

Riegel M. et al. (2009). Grammaire méthodique du français. Paris : PUF.

Vendler, Z. (1957). Verbs and times, repris dans Vendler, Z. (1967), Linguistics and philosophy. Ithace : Cornell University Press.

Wilmet, M. (2010). Grammaire critique du français (5e édition). Bruxelles : Duculot. 